\title{
Exercise-induced platelet and leucocyte activation is not enhanced in well-controlled Type 1 diabetes, despite increased activity at rest
}

\author{
H. Hu ${ }^{1}$ - B.-L. Johansson ${ }^{2}$ P. Hjemdahl ${ }^{1}$ N. Li $^{1}$ \\ ${ }^{1}$ Department of Medicine, Division of Clinical Pharmacology, Karolinska Hospital, Stockholm, Sweden \\ ${ }^{2}$ Department of Surgical Sciences, Division of Clinical Physiology, Karolinska Hospital, Stockholm, Sweden
}

\begin{abstract}
Aims/hypothesis. Stress can evoke a prothrombotic state with activated platelets and leucocytes, and increased platelet activation at rest has been reported for diabetic subjects. Thus, prothrombotic responses to stress may be enhanced in diabetes mellitus. We therefore evaluated platelet and leucocyte responses to exercise in Type 1 diabetic patients.

Methods. Type 1 diabetes mellitus patients with good metabolic control and healthy subjects matched for age and body mass index ( $n=15$ for both) performed a maximal exercise test. Platelet and leucocyte activation and their heterotypic aggregation were monitored by whole blood flow cytometry.

Results. Diabetic platelets did not show higher basal levels of P-selectin expression, but were more reactive to ADP and thrombin stimulation. Diabetic patients had increased lymphocyte and monocyte CD11b expression, and increased circulating platelet-monocyte aggregates. Exercise evoked thrombocytosis, increased circulating platelet $\mathrm{P}$-selectin expression, en-
\end{abstract}

hanced platelet sensitivity to ADP and thrombin, and elevated plasma levels of soluble P-selectin to a similar degree in diabetic patients and healthy subjects. Exercise induced marked leucocytosis and elevated plasma elastase, but only slightly increased leucocyte CD11b expression and leucocyte reactivity to stimulation by $\mathrm{N}$-formyl-methionyl-leucyl-phenylalanine. In all of these there was no difference between diabetic patients and healthy subjects. The numbers, but not percentages of circulating platelet-leucocyte aggregates were markedly increased by exercise, but the increase was less prominent among diabetic patients.

Conclusions/interpretation. Strenuous exercise evokes platelet and leucocyte activation in Type 1 diabetic patients and healthy subjects. Platelet and monocyte hyperactivity were found at rest, but responses to stress were not augmented in metabolically well-controlled Type 1 diabetes mellitus patients.

Keywords Leucocytes · Platelet-leucocyte aggregation $\cdot$ Platelets $\cdot$ Stress $\cdot$ Type 1 diabetes mellitus
Received: 23 October 2003 / Accepted: 26 January 2004

Published online: 17 April 2004

C) Springer-Verlag 2004

N. Li (®)

Department of Medicine, Division of Clinical Pharmacology,

Karolinska Hospital, 17176 Stockholm, Sweden

E-mail: Nailin.Li@ks.se

Tel.: +46-8-51773996, Fax: +46-8-331343

Abbreviations: FITC, fluorescein isothiocyanate .

fMLP, N-formyl-methionyl-leucyl-phenylalanine ·

MAb, monoclonal antibody $\cdot$ PLA, platelet-leucocyte aggregate $\cdot$ P-Lym, platelet-lymphocyte aggregate $\cdot$ P-Mon, platelet-monocyte aggregate $\cdot \mathrm{P}-\mathrm{Neu}$, platelet-neutrophil aggregate $\cdot$ RPE, R-phycoerythrin

\section{Introduction}

Diabetes mellitus is associated with an increased risk of cardiovascular morbidity and mortality, in which platelet and leucocyte dysfunction play important roles [1]. Thus, diabetic platelets are larger in size and express more adhesion molecules, such as glycoproteins IIb/IIIa and P-selectin [2, 3]. Platelets from diabetic patients are also hyperreactive to in vitro stimulation [4]. Diabetic leucocytes show increased elastase activity [5], enhanced adhesion molecule expression [6] and increased aggregability [7]. Other leucocyte functions, such as cell deformability [8], chemotaxis, 
and superoxide anion production [9] are also impaired in diabetic patients.

Stress may provoke cardiovascular events such as myocardial infarction [10], and responsiveness to stress may be of importance for cardiovascular risk. Exercise is a useful model in stress research [11, 12, 13], as it induces thrombocytosis and leucocytosis, activates platelets and leucocytes in vivo, potentiates their reactivity to in vitro stimuli and increases platelet-leucocyte heterotypic aggregation [11, 13]. Exercise also elevates coagulant factors, e.g. fibrinogen, FVIII and thrombin [12, 13, 14]. These changes may thus act in concert to promote a pro-thrombotic state during stress elicited by exercise.

Exercise induces more marked platelet activation in certain clinical settings, such as myocardial infarction [15]. Decreased prostacyclin production in response to strenuous exercise has been reported in Type 1 diabetic patients [16]. We thus hypothesised that exercise would elicit greater prothrombotic responses in Type 1 diabetes mellitus. We tested this hypothesis by comparing the effects of exercise on platelet and leucocyte activation in Type 1 diabetic patients with good metabolic control and matched healthy control subjects. Platelet and leucocyte activation ex vivo and reactivity to agonist stimulation in vitro were assessed by whole blood flow cytometry. Plasma levels of soluble P-selectin, elastase and von Willebrand factor were also measured to monitor platelet, leucocyte and endothelial activation respectively.

\section{Subjects and methods}

Subjects. Fifteen Type 1 diabetes mellitus patients and 15 healthy subjects gave informed consent to participate in the study, which was approved by the Ethics Committee of the Karolinska Institute.

Demographic variables and clinical characteristics are given in Table 1. The diabetic patients were in good metabolic control. Apart from mild background retinopathy in three patients, no other clinical signs of diabetic micro- and/or macroangiopathy were found. All subjects were non-smokers, had antecubital veins allowing clean venepunctures and stated that they had not taken aspirin or other platelet-active medication during the 2 weeks preceding the experiments.

Experiment procedures. All subjects had a light breakfast before coming to the laboratory for the exercise test, and were instructed not to drink beverages containing caffeine for at least 12 hours before the experiments. The patients also had their morning insulin injection. At 09.00 hours, the participants rested in the supine position for 30 minutes before pre-exercise blood sampling. After another 30 minutes of rest to allow processing of the resting samples, exercise was carried out on a computerised bicycle ergometer (Siemens-Elema AB, Solna, Sweden), with a starting workload of $30 \mathrm{~W}$ and increments of $10 \mathrm{~W} / \mathrm{min}$. Blood pressure and heart rate were monitored throughout the exercise process, and fatigue was estimated using the 20-grade Borg scale. Exercise was terminated upon exhaustion, i.e. at 19 to 20 on the Borg scale, and post-exercise blood samples were taken immediately afterwards.
Table 1. Characteristics of the subjects

\begin{tabular}{lcl}
\hline Characteristic & $\begin{array}{l}\text { Type 1 } \\
\text { Diabetes } \\
(n=15)\end{array}$ & $\begin{array}{l}\text { Healthy } \\
\text { subjects } \\
(n=15)\end{array}$ \\
\hline Age (years) & $31.8 \pm 1.8$ & $29.3 \pm 1.5$ \\
Disease duration (years) & $11 \pm 0.7$ & $\mathrm{NA}$ \\
BMI $\left(\mathrm{kg} / \mathrm{m}^{2}\right)$ & $24.4 \pm 0.7$ & $23.4 \pm 0.6$ \\
Blood glucose $(\mathrm{mmol} / \mathrm{l})$ & $9.6 \pm 1.3^{\mathrm{a}}$ & $5.1 \pm 0.2$ \\
$\mathrm{HbA}_{1} \mathrm{c}(\%)$ & $6.7 \pm 0.3^{\mathrm{a}}$ & $4.6 \pm 0.0$ \\
Plasma insulin $(\mu \mathrm{U} / \mathrm{ml})$ & $12.9 \pm 2.8$ & $13.0 \pm 1.1$ \\
Insulin dose $\left(\mathrm{U} \cdot \mathrm{kg}^{-1} \cdot \mathrm{day}^{-1}\right)$ & $0.7 \pm 0.1$ & $\mathrm{NA}$ \\
\hline
\end{tabular}

Results are means \pm SEM. a $p<0.05$, Type 1 diabetes mellitus patients compared to healthy subjects. NA, not applicable

Reagents. All antibodies were used at optimal concentrations, as determined by titration. Platelets were identified with fluorescein isothiocyanate (FITC)-conjugated anti-CD42a (GPIX) monoclonal antibody (MAb) Beb 1 (Becton Dickinson, San Jose, Calif., USA), and leucocytes were identified with R-phycoerythrin (RPE)-conjugated anti-CD45 MAb T29/33 (DAKO, Glostrup, Denmark). Platelet P-selectin expression was determined by RPE-CD62P MAb AC1.2 (Becton Dickinson). Leucocyte CD11b expression was determined by an FITC-conjugated MAb BEAR 1 (Immunotech, Marseille, France). FITC- and RPE-conjugated isotypic MAbs were used as negative controls. Fluorescent beads (Sphero Rainbow particles, 1.8-2.2 $\mu \mathrm{m})$ used for platelet counting were from PharMingen (San Diego, Calif., USA).

ADP, thrombin, N-formyl-methionyl-leucyl-phenylalanine (fMLP) and HEPES were from Sigma (St Louis, Mo., USA).

Flow cytometry. Venous blood was collected by venepuncture without stasis, using siliconised vacutainer tubes containing $1 / 10$ volume of $3.8 \%$ trisodium citrate (Becton Dickinson, Meylan, France). Five microlitre aliquots of blood were immediately added to HEPES-buffered saline (150 mmol/l $\mathrm{NaCl}$, $5 \mathrm{mmol} / \mathrm{l} \mathrm{KCl}, 1 \mathrm{mmol} / \mathrm{l} \mathrm{MgSO}_{4}, 10 \mathrm{mmol} / 1 \mathrm{HEPES}, \mathrm{pH} 7.4$ ) containing appropriately diluted antibodies in the absence or presence of the agonists. Samples were incubated at room temperature in the dark for $20 \mathrm{~min}$, and then diluted and mildly fixed with $0.5 \%$ formaldehyde saline.

Whole blood flow cytometric assays for measurements of platelet P-selectin expression [17], leucocyte CD11b expression [18], and platelet-leucocyte aggregates (PLAs) [17] have been described in detail previously. Platelet P-selectin expression data are reported as percentages of P-selectin-positive cells in the platelet population and as the absolute P-selectinpositive platelet count. Leucocyte CD11b expression is reported as mean fluorescence intensity of the total leucocyte population and leucocyte subpopulations. Platelet-leucocyte aggregates are presented as absolute counts and percentages of platelet-conjugated leucocytes in the total leucocyte population (i.e. PLA) and among lymphocytes (i.e. platelet-lymphocyte aggregates, P-Lym), monocytes (platelet-monocyte aggregates, P-Mon) and neutrophils (platelet-neutrophil aggregates, $\mathrm{P}-\mathrm{Neu})$.

Plasma preparation and immunoassays. Citrated blood was centrifuged (1400 $\mathrm{g}$ at $4{ }^{\circ} \mathrm{C}$ for 10 minutes) immediately after venepuncture. Plasma was aliquoted and stored at $-80{ }^{\circ} \mathrm{C}$ before analysis. Plasma levels of elastase (DPC Biermann, Bad Nauheim, Germany), soluble P-selectin (R\&D System, Abingdon, UK), von Willebrand factor (Diagnostica Stago, Asnières 
Table 2. Haematological measurements before and after exercise in Type 1 diabetes mellitus patients and healthy subjects

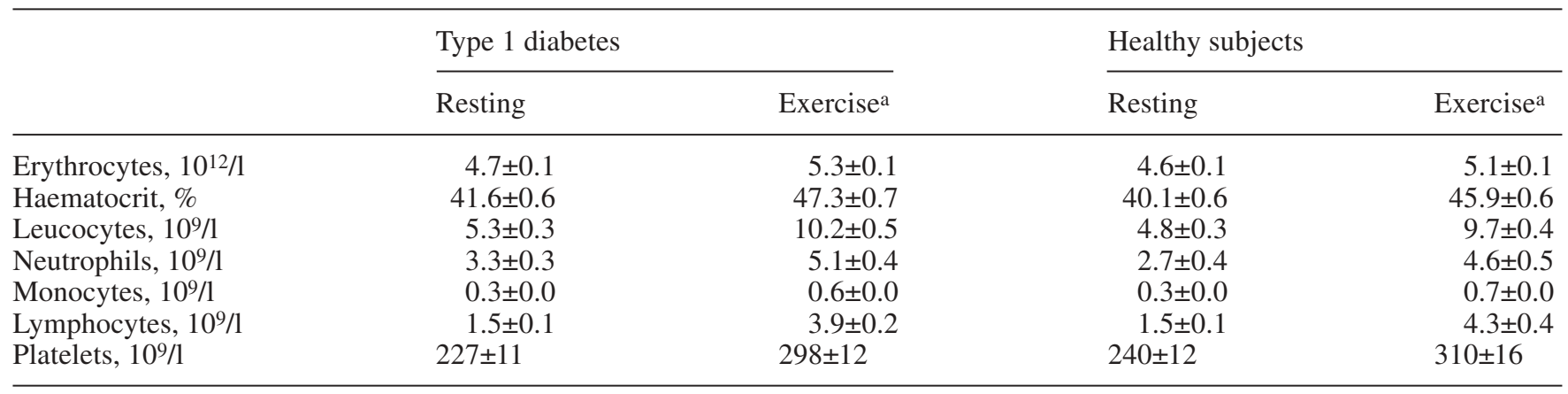

a $p<0.01$, exercise compared to resting values for all parameters

Cedex, France), and prothrombin fragment $1+2$ (F1+2; Behringwerke, Marburg, Germany) were determined by enzyme immunoassay. Catecholamine levels were determined by high-performance liquid chromatography as previously described [19].

Data presentation and statistics. Data are presented as means \pm SEM. Effects of exercise were analysed by repeated measures ANOVA and individual measurements were compared with Wilcoxon's signed rank test (STATISTICA, Tulsa, Okla., USA). A $p$ value of less than 0.05 was considered statistically significant.

\section{Results}

Physiological and haematological parameters. The maximal workload was $221 \pm 8 \mathrm{~W}$ in Type 1 diabetic patients and $249 \pm 12 \mathrm{~W}$ in healthy subjects $(p=0.07)$. Exercise increased (i) heart rate (from $63 \pm 2$ to $186 \pm 3$ beats per min in the patients and from $62 \pm 2$ to $196 \pm 4$ beats per $\min$ in the healthy subjects), (ii) systolic blood pressure (from $125 \pm 2$ to $198 \pm 3 \mathrm{mmHg}$ and from $116 \pm 3$ to $176 \pm 5 \mathrm{mmHg}$ respectively; $p<0.01$ for both), and (iii) blood cell counts (Table 2). Plasma norepinephrine increased from $1.2 \pm 0.1$ to $16.1 \pm 0.4 \mathrm{nmol} / \mathrm{l}$ in diabetic patients and from $2.3 \pm 0.6$ to $17.0 \pm 2.7 \mathrm{nmol} / \mathrm{l}$ in healthy subjects. Plasma epinephrine increased from $0.2 \pm 0.0$ to $2.4 \pm 0.9 \mathrm{nmol} / 1$ and from $0.2 \pm 0.0$ to $1.6 \pm 0.5 \mathrm{nmol} / 1$ respectively. Basal and post-exercise levels of catecholamines did not differ between the patients and healthy controls.

Platelet activation. The percentage of P-selectin-positive single platelets was not altered by exercise in the diabetic patients $(1.2 \pm 0.1 \%$ before and $1.2 \pm 0.7 \%$ after exercise), but the P-selectin-positive platelet count increased from $2.6 \pm 0.2 \times 10^{9} / 1$ to $3.6 \pm 0.2 \times 10^{9} / 1(p<0.001)$, due to exercise-induced thrombocytosis (Table 2). In healthy subjects, however, exercise increased the percentage of P-selectin-positive single platelets (from $1.0 \pm 0.1 \%$ to $1.6 \pm 0.2 \% ; p<0.01$ ), and the P-selectin-positive platelet count increased from $2.5 \pm 0.3 \times 10^{9} / 1$ to $4.9 \pm 0.8 \times 10^{9} / 1$. Neither P-selectin expression nor single platelet counts differed between
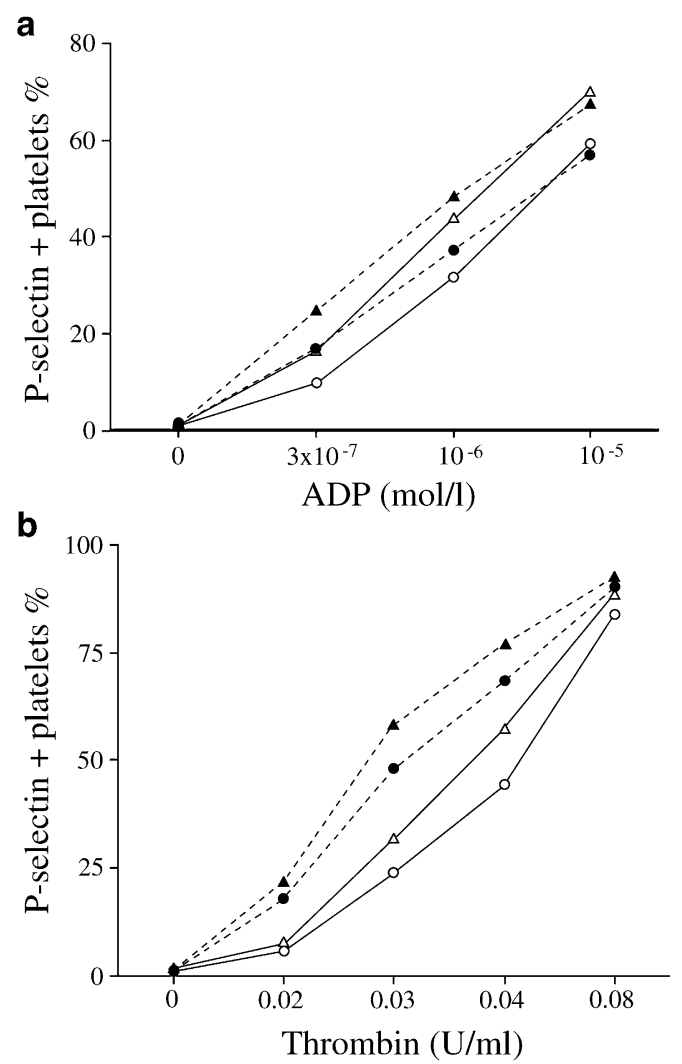

Fig. 1. Platelet reactivity to agonist stimulation before and after exercise. Blood samples were collected before (circles) and after (triangles) exercise from healthy subjects (open symbols, solid lines) and from Type 1 diabetic patients (filled symbols, dashed lines). Samples were incubated without or with increasing concentrations of $\operatorname{ADP}$ (a) or thrombin (b), and platelet P-selectin expression was determined by flow cytometry. Means from 15 subjects are plotted; $p$ values are from repeated measurements (ANOVA). Exercise enhanced platelet P-selectin expression in patients and healthy subjects $(p<0.01$ for both). Diabetic platelets had increased P-selectin expression upon stimulation with ADP $(p<0.01)$ or thrombin in vitro $(p<0.05)$ both before and after exercise

diabetes mellitus patients and healthy subjects before or after exercise.

As can be seen in Figure 1, both ADP and thrombin dose-dependently increased platelet P-selectin expres- 


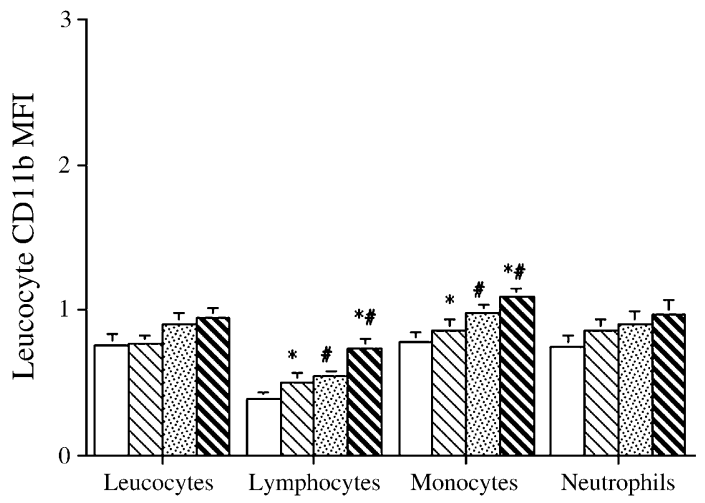

Fig. 2. Influence of exercise on leucocyte CD11b expression. Blood samples were obtained before and after exercise. Leucocyte CD11b expression was analysed by flow cytometry; CD11b mean fluorescent intensity (MFI) of pan leucocytes, lymphocytes, monocytes and neutrophils are presented as means \pm SEM. Healthy subjects before exercise: open bars; healthy subjects after exercise: light hatched bars; diabetic patients before exercise: dotted bars; diabetic patients after exercise: heavy hatched bars. $* p<0.01$, compared to before exercise; \# $p<0.01$, compared to corresponding data from healthy subjects

sion. Platelets from the diabetic patients were more sensitive to stimulation by ADP (Fig. 1a; ANOVA $p<0.01$ ) and thrombin (Fig. 1b; ANOVA $p<0.05$ ) than platelets from healthy subjects. However, exercise enhanced ADP- and thrombin-induced platelet activation to a similar degree in diabetic patients and healthy subjects.

Exercise elevated the plasma levels of soluble P-selectin from $53.0 \pm 3.7$ to $57.8 \pm 4.7 \mathrm{ng} / \mathrm{ml}$ in the diabetic patients and from $55.1 \pm 4.2$ to $66.8 \pm 6.3 \mathrm{ng} / \mathrm{ml}$ in healthy subjects $(p<0.001$ for both). Plasma-soluble P-selectin levels did not differ between diabetes mellitus patients and healthy subjects, either before or after exercise $(p=0.18)$.

Leucocyte activation. Compared to healthy subjects, leucocyte $\mathrm{CD} 11 \mathrm{~b}$ expression in the diabetic patients was slightly, albeit not significantly, higher among total leucocytes $(p=0.13)$ and neutrophils $(p=0.12)$, and significantly higher in monocytes and lymphocytes $(p<0.01$ for both) (Fig. 2).

Exercise increased leucocyte counts. The CD11b expression of monocytes and lymphocytes, but not of neutrophils, was elevated in the diabetic patients and in the healthy subjects after exercise $(p<0.05$ for both). The enhancement of leucocyte CD11b expression by exercise did not differ between diabetes mellitus patients and healthy subjects (ANOVA, $p=0.64$ ). Stimulation by fMLP induced marked leucocyte CD11b expression, which was slightly enhanced by exercise. For example, exercise increased fMLP-induced neutrophil CD11b expression from $2.23 \pm 0.15$ to $2.41 \pm 0.16$ in the diabetic patients $(p<0.05)$, and from $2.15 \pm 0.25$ to $2.30 \pm 0.11$ in healthy subjects $(p<0.01)$.
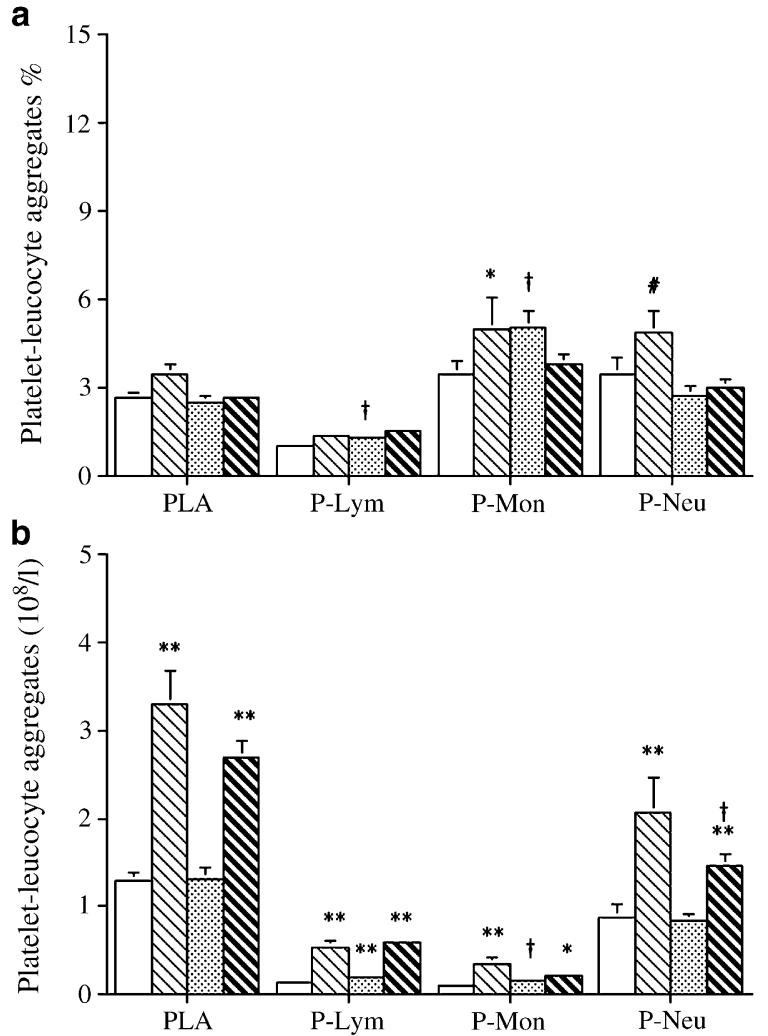

Fig. 3. Platelet-leucocyte aggregation before and after exercise. Whole blood samples were collected before and after exercise and analysed by flow cytometry. Percentages (a) and circulating quantities (b) of platelet-leucocyte aggregates (PLA), platelet-lymphocyte aggregates (P-Lym), plateletmonocyte aggregates (P-Mon) and platelet-neutrophil aggregates $(\mathrm{P}-\mathrm{Neu})$ are presented as means \pm SEM. Healthy subjects before exercise: open bars; healthy subjects after exercise: light hatched bars; diabetic patients before exercise: dotted bars; diabetic patients after exercise: heavy hatched bars. \# $p=0.07, * p<0.05, * * p<0.01$, compared to corresponding data before exercise; $\uparrow p<0.01$, compared to corresponding data from healthy subjects

These enhancements did not differ between the two groups (ANOVA, $p=0.84$ ).

Plasma elastase was elevated by exercise, from $25.6 \pm 1.8 \mathrm{ng} / \mathrm{ml}$ to $62.8 \pm 11.5 \mathrm{ng} / \mathrm{ml}$ in diabetic patients, and from $30 \pm 2.8 \mathrm{ng} / \mathrm{ml}$ to $67.0 \pm 6.7 \mathrm{ng} / \mathrm{ml}$ in healthy subjects $(p<0.001$ for both). No difference was found between patients and healthy subjects.

Platelet-leucocyte aggregation. The percentages of circulating PLAs at rest did not differ between diabetes mellitus patients and healthy subjects. However, when leucocyte subpopulations were analysed, circulating platelet-monocyte aggregates $(5.0 \pm 0.6 \%$ vs $3.4 \pm 0.4 \% ; p<0.01)$ and platelet-lymphocyte aggregates $(1.3 \pm 0.1 \%$ vs $1.0 \pm 0.1 \% ; p<0.01)$ were found to be higher in diabetes mellitus patients than in healthy subjects. Exercise had little influence on the percentages of circulating PLAs (Fig. 3a). Among leucocyte subpopulations, exercise slightly increased P-Mon 
$(p<0.05)$ and P-Neu percentages $(p=0.07)$ in the healthy subjects, but not among the diabetic patients (Fig. 3a). In contrast, circulating PLA counts were markedly increased after exercise in patients and healthy subjects $(p<0.001)$ (Fig. 3b). Increases were found in all leucocyte subpopulations in healthy subjects. The increases of PLA counts among diabetic patients were somewhat lower $(p=0.14)$, mainly due to a lesser increase of platelet-neutrophil aggregates $(p<0.01)$.

Other plasma parameters. Exercise increased von Willebrand factor from $1.13 \pm 0.04$ to $1.68 \pm 0.16 \mathrm{U}$ in diabetic patients and from $0.94 \pm 0.06$ to $1.65 \pm 0.10 \mathrm{U}$ in healthy subjects $(p<0.001$ for both). Thus, the patients had higher basal plasma levels of von Willebrand factor $(p<0.05)$, but there was no difference after exercise $(p=0.35)$.

Exercise increased prothrombin fragment $1+2$ from $0.47 \pm 0.02$ to $0.58 \pm 0.04 \mathrm{nmol} / \mathrm{l}$ in diabetic patients and from $0.54 \pm 0.04$ to $0.63 \pm 0.04 \mathrm{nmol} / \mathrm{l}$ in healthy subjects $(p<0.001$ for both; no difference between the groups, $p=0.61$ ).

\section{Discussion}

The present study shows that stress induced by strenuous exercise caused platelet and leucocyte activation in vivo, and enhanced their reactivity towards in vitro stimuli to a similar degree in Type 1 diabetes mellitus patients and healthy subjects. However, platelets from the Type 1 diabetes mellitus patients were hyperreactive to in vitro stimulation, in agreement with previous findings. In addition, CD11b expression of circulating monocytes was higher in diabetic patients before and after exercise.

Platelets from diabetes mellitus patients were hyperactive, as evidenced by platelet responses to ADP or thrombin stimulation in vitro and increased circulating platelet-monocyte aggregates, which reflect platelet activation in vivo. However, basal platelet Pselectin expression and soluble P-selectin were not increased among the diabetic patients, in agreement with the suggestion that platelet-monocyte aggregation is a more sensitive marker for platelet activation in vivo than single platelet P-selectin expression [20]. Mild leucocyte dysfunction was also present in our diabetic patients, as indicated by increased CD11b expression of circulating monocytes and enhanced monocyte $\mathrm{CD} 11 \mathrm{~b}$ expression after in vitro stimulation. Multiple mechanisms may account for diabetic platelet and leucocyte dysfunction. Thus, Type 1 diabetes mellitus is associated with enhanced protein kinase $\mathrm{C}$ activity [21], elevated platelet cytosolic $\mathrm{Ca}^{2+}$ levels due to increased $\mathrm{Ca}^{2+}$ ATPase activity [22], decreased nitric oxide synthase activity and increased superoxide anion production [22]. These alterations may act in concert to sensitise platelets and leucocytes.
Platelet activation during exercise is a complex issue. Exercise responses depend on exercise intensity and physical fitness of the tested subjects. It has been reported that moderate exercise may suppress platelet function, while intensive exercise (such as the exercise model used in the present study) primes and activates platelets $[13,14,23,24]$. There is also evidence that platelets from sedentary individuals are more sensitive to exercise than those from physically active ones [25].

Exercise (and other acute stressors) may trigger acute cardiovascular events [10], but increased fitness has a protective effect, which could be related to reduced platelet reactivity $[24,26,27]$. It should thus be stressed that chronic exercise may reduce longterm cardiovascular risk in diabetic patients via suppression of platelet responses [24, 26, 27] and that exercise may help to achieve good metabolic control in diabetic patients [28]. In the present study, otherwise healthy and physically fit Type 1 diabetes mellitus patients and healthy men matched for age and BMI were subjected to exhaustive exercise. Several mechanisms may contribute to exercise-enhanced platelet activation. Markedly elevated plasma catecholamines during exercise could prime platelets via alpha-2 adrenoceptor activation [29, 30]. Exercise increases cardiac output and blood pressure, and subsequently increases shear force in the blood stream, which may also activate platelets. In addition, exercise increases the generation of thrombin, which is a strong platelet agonist. Other factors possibly contributing to platelet activation are: exercise-enhanced agonist-induced $\mathrm{Ca}^{2+}$ influx [30], elevated plasma levels of elastase [31], and increased oxidative stress during exercise [32]. Although we confirmed our previous findings that strenuous exercise causes platelet activation in vivo and increases platelet reactivity in vitro [13], we did not find enhanced reactivity to exercise among the diabetic patients. Thus, the key mechanisms to strenuous exercise-induced platelet activation, which are not yet fully understood, are not specifically disturbed in Type 1 diabetes mellitus.

Exercise induces leucocytosis via leucocyte release from the bone marrow and demarginalisation of leucocytes from the spleen, the lungs and other organs. In agreement with our previous report [13], exercise enhanced leucocyte activation, as evidenced by increased CD11b expression and elevated plasma elastase levels. Exercise also enhanced leucocyte responsiveness to in vitro stimulation. Increased integrin expression of leucocytes facilitates their adhesion on and/or migration through endothelial cells, which are important processes in atherosclerosis. However, apart from increased platelet-monocyte aggregation at rest, we did not observe increased platelet-leucocyte aggregation in patients with Type 1 diabetes mellitus, either at rest or after exercise. 
The present study shows that exercise enhanced platelet and leucocyte activity, as well as platelet-leucocyte aggregation to a similar degree in Type 1 diabetic patients and healthy subjects. Our hypothesis that diabetes would be associated with enhanced responses to stress was thus not proven. However, our diabetic patients were physically fit and in good metabolic control, and had minimal or no signs of microvascular complications. Yet poor metabolic control is known to contribute to platelet and leucocyte dysfunction in Type 1diabetes mellitus [33, 34]. Our data thus suggest that alterations of platelet and leucocyte function are mild in well-controlled diabetes mellitus patients, and that good metabolic control may help maintain normal responses of platelets and leucocytes to stress, thus alleviating diabetic cardiovascular complications.

In conclusion, strenuous exercise induces multicellular activation in vivo and promotes a prothrombotic state in Type 1 diabetes mellitus patients and healthy subjects. Diabetic platelets and monocytes were hyperactive, but the hyperactivity was not further augmented by stress. Thus, well-controlled Type 1 diabetes mellitus may not sensitise patients to the risk of cardiovascular events triggered by stress.

Acknowledgements. This study was supported by grants from the Swedish Heart-Lung Foundation, the Swedish Research Council (5930), the Swedish Medical Association, the Stockholm County Council, and the Karolinska Institute. The authors are grateful for the expert technical assistance of Maud Daleskog, Maj-Christina Johansson, Alice Skogholm, Agneta Reinholdsson and Eva-Lena Forsberg.

\section{References}

1. Biondi-Zoccai GG, Abbate A, Liuzzo G, Biasucci LM (2003) Atherothrombosis, inflammation, and diabetes. J Am Coll Cardiol 41:1071-1077

2. Tschoepe D, Roesen P, Esser J et al. (1991) Large platelets circulate in an activated state in diabetes mellitus. Semin Thromb Hemost 17:433-438

3. Tschoepe D, Driesch E, Schwippert B, Nieuwenhuis HK, Gries FA (1995) Exposure of adhesion molecules on activated platelets in patients with newly diagnosed IDDM is not normalized by near-normoglycemia. Diabetes 44: 890-894

4. Winocour PD (1994) Platelets, vascular disease, and diabetes mellitus. Can J Physiol Pharmacol 72:295-303

5. Collier A, Jackson M, Bell D et al. (1989) Neutrophil activation detected by increased neutrophil elastase activity in type 1 (insulin-dependent) diabetes mellitus. Diabetes Res 10:135-138

6. Olson JA, Whitelaw CM, McHardy KC, Pearson DW, Forrester JV (1997) Soluble leucocyte adhesion molecules in diabetic retinopathy stimulate retinal capillary endothelial cell migration. Diabetologia 40:1166-1171

7. Elhadd TA, Bancroft A, McLaren M, Newton RW, Belch JJ (1997) Increased granulocyte aggregation in vitro in diabetes mellitus. QJM 90:461-464

8. Linderkamp O, Ruef P, Zilow EP, Hoffmann GF (1999) Impaired deformability of erythrocytes and neutrophils in children with newly diagnosed insulin-dependent diabetes mellitus. Diabetologia 42:865-869

9. Wierusz-Wysocka B, Wykretowicz A, Byks H, Sadurska K, Wysocki H (1993) Polymorphonuclear neutrophils adherence, superoxide anion $\left(\mathrm{O}_{2}^{-}\right)$production and HBA1 level in diabetic patients. Diabetes Res Clin Pract 21:109-114

10. Willich SN, Lewis M, Löwel H, Arntz H-R, Schubert F, Schröder R (1993) Physical exertion as a trigger of acute myocardial infarction. N Engl J Med 329:1684-1690

11. Kestin AS, Ellis PA, Barnard MR, Errichetti A, Rosner BA, Michelson AD (1993) Effect of strenuous exercise on platelet activation state and reactivity. Circulation 88 : 1502-1511

12. El-Sayed MS, Sale C, Jones PG, Chester M (2000) Blood hemostasis in exercise and training. Med Sci Sports Exerc 32:918-925

13. Li N, Wallen NH, Hjemdahl P (1999) Evidence for prothrombotic effects of exercise and limited protection by aspirin. Circulation 100:1374-1379

14. Wallen NH, Goodall AH, Li N, Hjemdahl P (1999) Activation of haemostasis by exercise, mental stress and adrenaline: effects on platelet sensitivity to thrombin and thrombin generation. Clin Sci 97:27-35

15. Hurlen M, Seljeflot I, Arnesen H (2000) Increased platelet aggregability during exercise in patients with previous myocardial infarction. Lack of inhibition by aspirin. Thromb Res 99:487-494

16. Koivisto VA, Jantunen M, Sane T et al. (1989) Stimulation of prostacyclin synthesis by physical exercise in type I diabetes. Diabetes Care 12:609-614

17. Li N, Goodall AH, Hjemdahl P (1999) Efficient flow cytometric assay for platelet-leukocyte aggregates in whole blood using fluorescence signal triggering. Cytometry 35 : 154-161

18. Li N, Hallden G, Hjemdahl P (2000) A whole-blood flow cytometric assay for leukocyte CD11b expression using fluorescence signal triggering. Eur J Haematol 65:57-65

19. Hjemdahl P (1987) Catecholamine measurements in plasma by high-performance liquid chromatography with electrochemical detection. Methods Enzymol 142:521-534

20. Michelson AD, Barnard MR, Krueger LA, Valeri CR, Furman MI (2001) Circulating monocyte-platelet aggregates are a more sensitive marker of in vivo platelet activation than platelet surface P-selectin: studies in baboons, human coronary intervention, and human acute myocardial infarction. Circulation 104:1533-1537

21. Kunt T, Forst T, Fruh B et al. (1999) Binding of monocytes from normolipidemic hyperglycemic patients with type 1 diabetes to endothelial cells is increased in vitro. Exp Clin Endocrinol Diabetes 107:252-256

22. Levy J, Gavin JR 3rd, Sowers JR (1994) Diabetes mellitus: a disease of abnormal cellular calcium metabolism? Am J Med 96:260-273

23. El-Sayed MS (1996) Effects of exercise on blood coagulation, fibrinolysis and platelet aggregation. Sports Med 22: 282-298

24. Wang JS, Jen CJ, Kung HC, Lin LJ, Hsiue TR, Chen HI (1994) Different effects of strenuous exercise and moderate exercise on platelet function in men. Circulation 90:28772885

25. Cuzzolin L, Lussignoli S, Crivellente F et al. (2000) Influence of an acute exercise on neutrophil and platelet adhesion, nitric oxide plasma metabolites in inactive and active subjects. Int J Sports Med 21:289-293

26. Wang JS, Jen CJ, Chen HI (1995) Effects of exercise training and deconditioning on platelet function in men. Arterioscler Thromb Vasc Biol 15:1668-1674 
27. Wang JS, Jen CJ, Chen HI (1997) Effects of chronic exercise and deconditioning on platelet function in women. J Appl Physiol 83:2080-2085

28. Castaneda C, Layne JE, Munoz-Orians L et al. (2002) A randomized controlled trial of resistance exercise training to improve glycemic control in older adults with type 2 diabetes. Diabetes Care 25:2335-2341

29. Larsson PT, Wallen NH, Egberg N, Hjemdahl P (1992) Alpha-adrenoceptor blockade by phentolamine inhibits adrenaline-induced platelet activation in vivo without affecting resting measurements. Clin Sci 82:369-376

30. Wang JS, Cheng LJ (1999) Effect of strenuous, acute exercise on alpha2-adrenergic agonist-potentiated platelet activation. Arterioscler Thromb Vasc Biol 19:1559-1565
31. Renesto P, Chignard M (1993) Enhancement of cathepsin G-induced platelet activation by leukocyte elastase: consequence for the neutrophil-mediated platelet activation. Blood 82:139-144

32. Tozzi-Ciancarelli MG, Penco M, Di Massimo C (2002) Influence of acute exercise on human platelet responsiveness: possible involvement of exercise-induced oxidative stress. Eur J Appl Physiol 86:266-272

33. Marhoffer W, Stein M, Maeser E, Federlin K (1992) Impairment of polymorphonuclear leukocyte function and metabolic control of diabetes. Diabetes Care 15:256-260

34. Pellegatta F, Folli F, Ronchi P, Caspani L, Galli L, Vicari AM (1993) Deranged platelet calcium homeostasis in poorly controlled IDDM patients. Diabetes Care 16:178-183 